\title{
Phase unwrapping algorithm using polynomial phase approximation and linear Kalman filter
}

\author{
Rishikesh Kulkarni ${ }^{1, *}$ and Pramod Rastogi ${ }^{2}$ \\ ${ }^{1}$ Department of Electronics and Electrical Engineering, Indian Institute of Technology Guwahati, India \\ ${ }^{2}$ Applied Computing and Mechanics laboratory, Ecole Polytechnique Federale de Lausanne, Switzerland \\ *Corresponding author: rishi.k@iitg.ernet.in
}

Compiled December 29, 2017

\begin{abstract}
A noise robust phase unwrapping algorithm is proposed based on state space analysis and polynomial phase approximation using wrapped phase measurement. The true phase is approximated as a two-dimensional first order polynomial function within a small sized window around each pixel. The estimates of polynomial coefficients provide the measurement of phase and local fringe frequencies. A state space representation of spatial phase evolution and the wrapped phase measurement is considered with the state vector consisting of polynomial coefficients as its elements. Instead of using the traditional nonlinear Kalman filter for the purpose of state estimation, we propose to use the linear Kalman filter operating directly with the wrapped phase measurement. Adaptive window width is selected at each pixel based on the local fringe density to strike a balance between the computation time and noise robustness. In order to retrieve the unwrapped phase, either a line scanning approach or quality guided strategy of pixel selection is used depending on the underlying continuous or discontinuous phase distribution, respectively. Simulation and experimental results are provided to demonstrate the applicability of the proposed method. () 2017 Optical Society of America
\end{abstract}

OCIS codes: (120.5050) Phase measurement; (110.2650) Fringe analysis; (120.6160) Speckle interferometry.

http://dx.doi.org/10.1364/ao.XX.XXXXXX

\section{INTRODUCTION}

Phase unwrapping is of prime importance in various measurement techniques such as holographic interferometry [1], electronic speckle pattern interferometry [2], synthetic aperture radar interferometry [3], magnetic resonance imaging [4] and fringe projection profilometry [5]. In these techniques, information on the measurand is encoded in the form of a two-dimensional phase. However, the arctangent operation involved in these techniques provides the phase measurement in a wrapped form, i.e., the phase values are mapped in the range of $(-\pi, \pi]$. The measurand, however, is proportational to the true phase which makes phase unwrapping an indispensable task. The relationship between true phase $\psi$ and wrapped phase $\phi$ is given as

$$
\begin{aligned}
\phi(x, y) & =\mathscr{W}(\psi(x, y)) \\
& =\bmod (\psi(x, y)+\pi, 2 \pi)-\pi
\end{aligned}
$$

where, $\mathscr{W}(\cdot)$ represents the wrap operator which maps the phase $\psi$ in the range $(-\pi, \pi]$. Note that $\phi$ represents the actual wrapped measurement of the true phase. Two-dimensional distribution of $\phi$ is usually termed as phase fringe pattern. In ideal conditions, i.e., in the absence of any noise, phase unwrapping is trivial if continuous phase distribution is assumed. A simple unwrapping algorithm provides the unwrapped phase estimate by performing appropriate addition/subtraction of integer multiple of $2 \pi$ at each pixel depending on the phase difference between the neighboring pixels. The estimated phase is independent of the phase unwrapping path in such cases. However, if the true phase distribution inherently consists of discontinuities, a path-following phase unwrapping strategy has to be adapted. Moreover, in practice, the wrapped phase is usually corrupted by various noise sources. A wrongly chosen phase unwrapping path may lead to error propagation and ultimately errorneous phase estimate. These practical limitations make phase unwrapping a non-trivial operation. Consequently, a lot of research on various phase unwrapping strategies and algorithms have been reported over the years $[6,7]$. A noise-robust algorithm to evaluate spatial frequencies on fringe patterns based on statistical image processing was described in Ref. [8] for the numerical correction of an optical vortex present in the phase fringe pattern. Some of the popular phase unwrapping methods can be given as branch-cut [9-12], quality guided [13-16] and least-square algorithms [17-21]. While some of them are robust to certain level of noise, most of them require phase fringe pattern denoising [22-24] as a pre-processing operation.

In recent years, a number of alogrithms have been reported which simultaneously perform noise filtering and phase unwrapping operation [25]. The progagation of phase estimation error associated with independent filtering and unwrapping operations is avoided in such algorithms. In some of these methods, the problem of phase unwrapping is formulated in the form of state space analysis. The spatial evolution 
of phase and measurements are given by a state space representation. Extended Kalman filter (EKF) [26, 27] and unscented Kalman filter (UKF) [28-30] have been the main choices for the purpose of state estimation. The proposed algorithm falls under this catergory of phase unwrapping methods. However, instead of the conventional use of nonlinear Kalman filters mentioned above, we propose to use linear Kalman filter along with local first order polynomial phase approximation.

\section{ALGORITHM}

\section{A. Local Polynomial Approximation of Phase}

Different phase unwrapping algorithms have been proposed based on segmentwise [31-33] or blockwise [34-36] polynomial phase approximation. Although these methods provide satisfactory results in some cases, they are based on two important assumptions that require first, the true phase distribution to be spatially continuous and second, the wrapped phase measurement to be available over the entire segment or block. However, these assumptions are somewhat restrictive in practice. For example, in some cases, a certain portion of the phase fringe pattern may need to be masked during the phase unwrapping process in order to bypass an occluded or low intensity area on the object surface. At such locations, lack of fringe measurement may cause inaccurate phase unwrapping which may propagate in the valid fringe regions. The assumption of continuous phase distribution allows one to perform the phase unwrapping in either row-by-row or column-by-column manner, that is, using a line scanning approach. However, this approach cannot provide a reliable phase estimate if the true phase contains inherent discontinuities such as step changes. Furthermore, the presence of noise may cause false indication of absolute phase jumps greater than $\pi$ between two neighboring pixels.

Recently, we have proposed a noise robust phase unwrapping algorithm [37] based on first order polynomial phase approximation within a small size window around each pixel. This pixelwise phase unwrapping method is capable of providing the unwrapped phase estimate from a highly noisy phase fringe pattern which may contain masked regions. However, the restrictive assumption of continuous phase impairs the method in providing phase estimate containing discontinuities. In this paper, we consider a similar model of local phase by approximating the true phase around each pixel as a two-dimensional first order polynomial function of spatial coordinates. That is,

$$
\psi_{L}\left(x_{L}, y_{L}\right)=c_{0}+c_{1} x_{L}+c_{2} y_{L},
$$

where, $x_{L} \in[-L, L]$ and $y_{L} \in[-L, L]$ represent the spatial coordinates within a window around the pixel $(x, y)$ such that $\psi(x, y)=\psi_{L}(0,0)=$ $c_{0} ; L$ represents the window width; and $\left[c_{0}, c_{1}, c_{2}\right]$ represents the set of polynomial coefficients. The unwrapped phase estimate at $(x, y)$ is obtained using the wrapped phase measurement available within the analysis window. In fact, a similar polynomial phase model has been considered in phase unwrapping methods proposed in $[38,39]$. A fixed window width is used in [38] and adaptive window width is evaluated at each pixel from a pre-defined set of values in the PhaseLa method [39]. In these techniques, a nonlinear least square method of polynomial coefficient estimation is applied in a sliding window manner to obtain continuous unwrapped phase distribution. On the other hand, the method proposed in [37] utilizes state space analysis in a sliding window manner to estimate polynomial coefficients at each pixel.

\section{B. Polynomial Coefficient Estimation Using State Space Anal- ysis}

A state space analysis based polynomial coefficient estimation algorithm similar to the one proposed in [37] is described in this paper as follows:

$$
\begin{aligned}
& \boldsymbol{C}_{l}=\boldsymbol{F C}_{l-1}, \\
& \Gamma_{l}=\phi_{L}\left(x_{L}, y_{L}\right)
\end{aligned}
$$

where, $\boldsymbol{C}=\left[c_{0}, c_{1}, c_{2}\right]^{T}$ represents the state vector containing polynomial coefficients as its elements. The fringe within analysis window consists a total of $(2 L+1)^{2}$ meausurement samples which are scanned in a row-by-row manner for state vector updation. Accordingly, the variable $l$ takes values within the range $\left[1,(2 L+1)^{2}\right]$. Equation (2) represents the state transition model with transition matrix $\boldsymbol{F}$. The state estimation is performed within the analysis window utilizing the wrapped phase measurement $\phi_{L}\left(x_{L}, y_{L}\right)$ defined such that $\phi(x, y)=\phi_{L}(0,0)$. Since the polynomial coefficients are defined to be constant within the analysis window in accordance to Eq. (1), the state transition model does not bring any change in the state vector. Consequently, $\boldsymbol{F}$ is defined to be an identity matrix of size $3 \times 3$. The major distinction between the state space model considered in [37] and in this paper is the definition of measurement vector. Whereas a $2 \times 1$ sized vector $\Gamma_{l}=\left[\cos \left(\phi_{L}\left(x_{L}, y_{L}\right)\right), \sin \left(\phi_{L}\left(x_{L}, y_{L}\right)\right)\right]$ is considered in [37], we consider a single element vector as given in Eq. (3). Since we have, $\cos \left(\psi_{L}\left(x_{L}, y_{L}\right)\right)=\cos \left(\phi_{L}\left(x_{L}, y_{L}\right)\right)$ and $\sin \left(\psi_{L}\left(x_{L}, y_{L}\right)\right)=\sin \left(\phi_{L}\left(x_{L}, y_{L}\right)\right)$, a nonlinear measurement model is considered in most of the state space analysis based methods. Accordingly, the $\Gamma_{l}$ is modelled as a nonlinear function of state vector in the presence of additive noise. On the other hand, in the prosposed method the measurement is modelled as a function of state vector as follows,

$$
\Gamma_{l}=\mathscr{W}\left(\boldsymbol{H}_{l} \boldsymbol{C}_{l}\right)+\varepsilon_{l},
$$

where $\boldsymbol{H}_{l}=\left[\begin{array}{lll}1 & x_{L} & y_{L}\end{array}\right] ; \varepsilon_{l}$ represents the additive white Gaussian noise of zero mean and variance $R_{l}$.

It is important to note that the term $\boldsymbol{H}_{l} \boldsymbol{C}_{l}$ essentially represents the true phase $\psi_{L}\left(x_{L}, y_{L}\right)$. Since the phase is considered to be corrupted by white Gaussian noise, we can treat $\psi$ as a random variable. We have the measurement $\phi$ of this random variable such that $\phi=\mathscr{W}(\psi)$. Considering normal distribution of $\psi=\mathscr{N}\left(\mu, \sigma^{2}\right)$, the wrapped Gaussian distribution of $\phi$ is obtained as [40],

$$
P\left(\phi ; \mu, \sigma^{2}\right)=\sum_{k=-\infty}^{\infty} \frac{1}{\sqrt{2 \pi \sigma^{2}}} \exp \left[-\frac{(\phi-(\mu+2 \pi k))}{2 \sigma^{2}}\right] .
$$

Recently, a design of wrapped Kalman filter based on circular data statistics has been proposed [41] as an alternative to the EKF and the UKF for non-linear dynamic systems. It has been shown that the linear Kalman filter with one dimensional measurement $\left(\Gamma_{l}=\right.$ $\left.\phi_{L}\left(x_{L}, y_{L}\right)\right)$ and wrapped phase statistics in Eq. (5) can provide better accuracy in state estimation compared to the EKF or UKF with the measurement vector $\left(\Gamma_{l}=\left[\cos \left(\phi_{L}\left(x_{L}, y_{L}\right)\right), \sin \left(\phi_{L}\left(x_{L}, y_{L}\right)\right)\right]\right)$. A one dimensional phase unwrapping algorithm has been proposed utilizing this wrapped Kalman filter [42] based on assumption of continuous phase distribution. We propose a linear Kalman filter based algorithm of state estimation for the purpose of phase unwrapping as follows [43]:

1. Initialize the state vector estimate at $l=0$ and its error covariance matrix as

$$
\begin{aligned}
& \hat{\boldsymbol{C}}_{0}^{+}=\mathbb{E}\left[\boldsymbol{C}_{0}\right], \\
& \boldsymbol{P}_{0}^{+}=\mathbb{E}\left[\left(\boldsymbol{C}_{0}-\hat{\boldsymbol{C}}_{0}^{+}\right)\left(\boldsymbol{C}_{0}-\hat{\boldsymbol{C}}_{0}^{+}\right)^{T}\right],
\end{aligned}
$$

where, $\mathbb{E}$ is the expectation operator. The superscripts - and + indicate the a priori and a posteriori estimates of the associated variables. 
2. Since the state vector remains unchanged during the prediction operation at the $l$ th step, set

$$
\begin{aligned}
& \hat{\boldsymbol{C}}_{l}^{-}=\hat{\boldsymbol{C}}_{l-1}^{+}, \\
& \boldsymbol{P}_{l}^{-}=\boldsymbol{P}_{l-1}^{+} .
\end{aligned}
$$

3. Compute the unwrapped phase estimate using the predicted state vector as

$$
\hat{\Gamma}_{l}=\boldsymbol{H}_{l} \hat{\boldsymbol{C}}_{l}^{-}
$$

4. Perform the measurement update of the state vector as

$$
\begin{aligned}
\boldsymbol{K}_{l} & =\boldsymbol{P}_{l}^{-} \boldsymbol{H}_{l}^{T}\left(\boldsymbol{H}_{l} \boldsymbol{P}_{l}^{-} \boldsymbol{H}_{l}^{T}+R_{l}\right)^{-1}, \\
\hat{\boldsymbol{C}}_{l}^{+} & =\hat{\boldsymbol{C}}_{l}^{-}+\boldsymbol{K}_{l} \mathscr{W}\left(\Gamma_{l}-\hat{\Gamma}_{l}\right), \\
\boldsymbol{P}_{l}^{+} & =\left(\boldsymbol{I}-\boldsymbol{K}_{l} \boldsymbol{H}_{l}\right) \boldsymbol{P}_{l}^{-}\left(\boldsymbol{I}-\boldsymbol{K}_{l} \boldsymbol{H}_{l}\right)^{T}+\boldsymbol{K}_{l} R_{l} \boldsymbol{K}_{l}^{T},
\end{aligned}
$$

where, $\boldsymbol{K}_{l}$ is Kalman gain. Note that the error between the estimated unwrapped phase and measured wrapped phase is wrapped prior to the measurement update of state vector estimate. The proposed method of state estimation differs from that used in the design of wrapped Kalman filter. In [41], upto three replicas of the measurement are used in the state vector updation. That is,

$$
\left.\hat{\boldsymbol{C}}_{l}^{+}=\hat{\boldsymbol{C}}_{l}^{-}+\boldsymbol{K}_{l} \sum_{k=-1}^{k=1}\left[\left(\Gamma_{l}+2 \pi k\right)-\hat{\Gamma}_{l}\right)\right] \eta_{l, k},
$$

where,

$$
\eta_{l, k}=\frac{\mathscr{N}\left(\Gamma_{l}+2 \pi k ; \hat{\Gamma}_{l}, R_{l}\right)}{\sum_{m=-1}^{m=1} \mathscr{N}\left(\Gamma_{l}+2 \pi m ; \hat{\Gamma}_{l}, R_{l}\right)} .
$$

It should be noted that the main aim in [41] was to accurately compute the wrapped estimate of the state vector. In order to do so, it is required to wrap the predicted state vector estimate prior to the measurement update step. On the other hand, the difference of estimated (unwrapped) and observed (wrapped) phase (See Eq. (12)) should be wrapped to obtain reliable estimate of the state vector in the measurement update step in the proposed method.

Figure 1 shows an example to demonstrate the direct use of wrapped phase measurement in Kalman filter based state estimation. The true phase and its wrapped form are shown in Fig. 1(a). The high magnitude difference between the unwrapped phase and wrapped phase shown in Fig. 1(b) is prone to be wrongly interpreted in Eq. (12) by the Kalman filter and which can result in inaccurate polynomial coefficient estimation. However, if this difference is wrapped prior to the measurement update of the state estimate, the state estimation is performed accurately on account of small magnitude of the wrapped difference shown in Fig. 1(b).

\section{Adaptive Window Width Selection}

We have seen that the coefficient estimate $\hat{c}_{0}$ at $l=(2 L+1)^{2}$ provides the unwrapped phase estimate at pixel $(x, y)$. At the same time, the coefficient estimates $\hat{c}_{1}$ and $\hat{c}_{2}$ provide the local fringe frequencies in function of $x$ and $y$, respectively. The total local frequency (TLF) can be defined as

$$
T L F(x, y)=\sqrt{\hat{c}_{1}^{2}+\hat{c}_{2}^{2}}
$$

Typically, it is suggested [39] that small and high window widths should be used while analyzing high and low density fringe areas, respectively. This is done to ensure that the local fringe frequencies are estimated accurately which in turn provide accurate unwrapped phase estimation. As a matter of fact, in speckle metrology, the phase noise is higher in

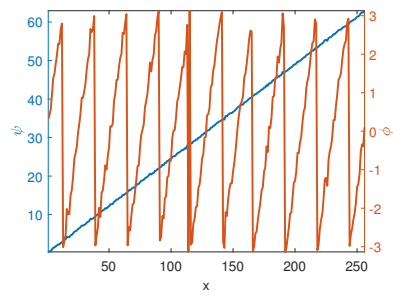

(a)

Fig. 1. (a) Simulated $\psi$ and $\phi$ (in radians) and (b) unwrapped and wrapped difference $(\psi-\phi)$.

high density fringe areas compared to that in low density fringe areas [44]. Consequently, the use of a small window width at high density fringe areas provides phase estimate with lower bias at the expense of increased noise sensitivity due to insufficient fringe measurement samples. We propose to utilize adaptive window width selection in the process of state estimation at each pixel. The adaptive window width is selected from a pre-defined set of values based on the value of the TLF computed at the already unwrapped pixel and which is used in the initialization step (Eq. (6)) of the current pixel. We empirically select the adaptive window width based on the following criterion:

$$
L_{\text {adaptive }}(x, y)= \begin{cases}1, & \text { if } T L F<0.75 \\ 2, & \text { else if } T L F<1 \\ 3, & \text { else if } T L F<1.5 \\ 4, & \text { otherwise }\end{cases}
$$

It is important to note that in [39], the polynomial coefficients are required to be estimated for each value of window width from the predefined set in order to compute the adaptive window width at each pixel. On the other hand, in the proposed method, the adaptive window width is selected based on the value of the TLF computed at the initilalizing neighbor pixel.

\section{Phase Quality Map And Guiding Strategy}

As mentioned earlier, phase discontinuities and noise sources can cause error in unwrapped phase estimation if path indepdendent fringe scanning is performed. Accordingly, a number of phase quality maps and unwrapping path guiding strategies have been reported in the literature [13]. Whereas the phase quality maps decide accuracy with which the unwrapped phase can be estimated, the path guiding strategies determine the computational effeciency of phase unwrapping operation. A comparison of number of phase quality maps, such as, phase derivative variance, phase difference and transform based quality maps has been provided in [13]. Considering the measurement of wrapped phase alone, we propose to use phase derivative variance based phase quality map along with the classical guiding strategy of operating at high quality pixels with higher priority over low quality pixels. The choice of qualily maps and an unwrapping path guiding strategy may depend on the optical measurement setup.

The flow chart of the proposed phase unwrapping algorithm is provided in Appendix for readers' convenience.

\section{SIMULATION AND EXPERIMENTAL RESULTS}

Figure 2(a) shows simulated true phase map of size $384 \times 384$. The phase values are in radians. A noisy wrapped phase shown in Fig. 2 (b) is derived with the noise variance $\sigma_{\varepsilon}=0.7$. As the phase is continuous throughout the frame, the phase unwrapping is performed 


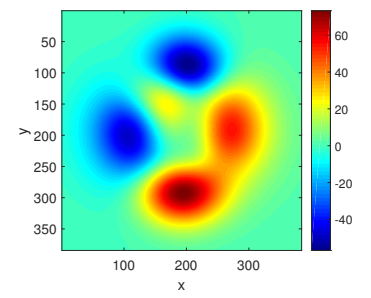

(a)

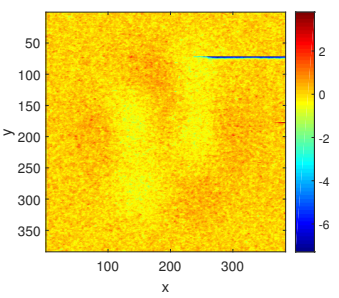

(c)

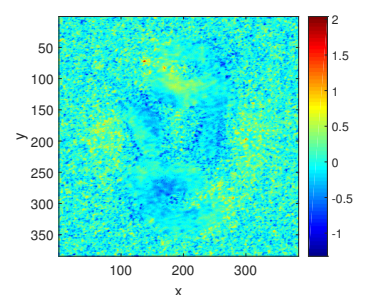

(e)

(f)

Fig. 2. (a) Simulated true phase (b) noisy wrapped phase simulated with $\sigma_{\varepsilon}=0.7$. Errors in the unwrapped phase estimation in radians computed using (c) linear Kalman filter with fixed window $L=1$, (d) linear Kalman filter with adpative window, (e) extended Kalman filter with adpative window, and (f) the PhaseLa method.

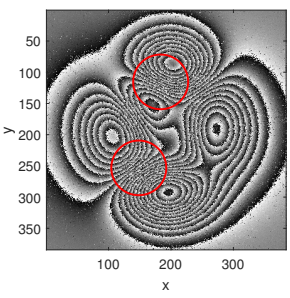

(a)

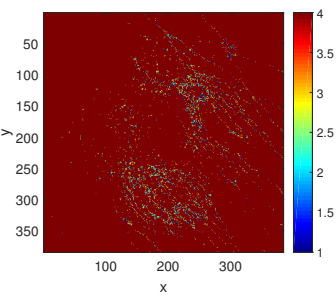

(c)

Fig. 3. (a) Speckle noise corrupted phase fringe pattern. Maps of adaptive window width corresponding to Fig. 3(a) computed using (b) the proposed method and (c) the PhaseLa method. (d) Rewrapped phase estimate computed using the proposed method.

using both LKF and EKF with the line-scanning approach. Figures 2(c) and (d) show the error in the unwrapped phase estimates computed

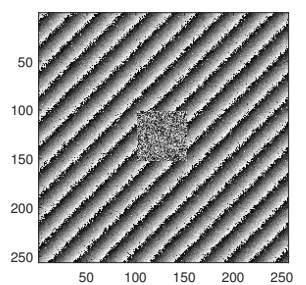

(a)

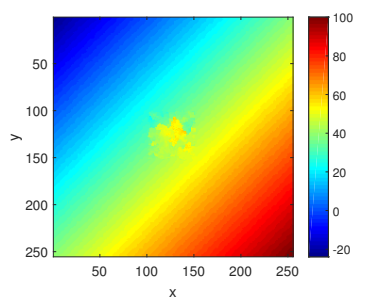

(c)

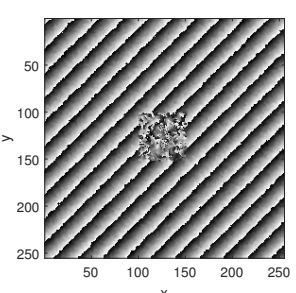

(e)

Fig. 4. Simulated phase fringe patterns with (a) a random phase noise region, and (b) a true phase discontinuity. (c) and (d) Unwrapped phase estimates computed from the phase fringe patterns shown in (a) and (b), respectively, using the proposed method. (e) and (f) Re-wrapped phase maps corresponding to (c) and (d), respectively. All the phase values are in radians.

using LKF with fixed window width $L=1$ and adpative window width selected from $L=[1,2,3,4]$, respectively. The use of fixed window resulted in an errorneous phase estimate due to the error propagation as observed in Fig. 2(c). On the other hand, the selected adaptive window width avoided such error propagation and provided accurate phase estimate. This result demonstrates the higher noise robustness offered by the adaptive window width selection strategy over the fixed window width strategy in phase unwrapping. Errors in the unwrapped phase estimation computed using the EKF based method with adaptive window width selection and the PhaseLa method are shown in Figs. 2(e) and (f), respectively. Although the accuracy offered by LKF and EKF based methods are equivalent, the LKF based method is found to be two times faster than the EKF based method. On the other hand, the PhaseLa method failed to perform accurate phase unwrapping.

A more realistic simulation of a speckle noise corrupted phase fringe pattern is performed using the method proposed in [44]. Accordingly, the phase decorrelation noise is predominant in the high fringe density area compared to the low fringe density area as indicated in Fig. 3(a). Two contrary views exist on the selection of adaptive window width in the PhaseLa method and the proposed method. Whereas a small(wide) window width is selected at high(low) fringe density pixel in the PhaseLa method, a wide(small) window width is selected in the proposed method. Both the methods are implemented with the set $L=[1,2,3,4]$ for the adaptive window width to select from. The maps of window widths selected at each pixel in the proposed method and the PhaseLa method are shown in Fig. 3(b) and (c), respectively. 


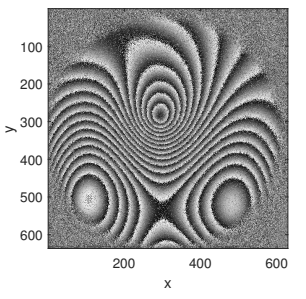

(a)

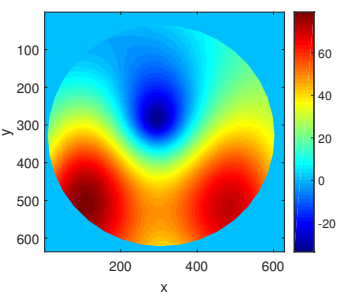

(c)

(d)

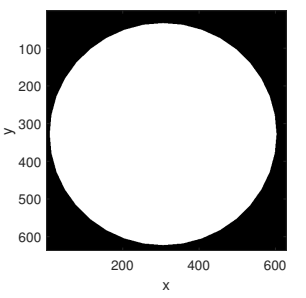

(b)

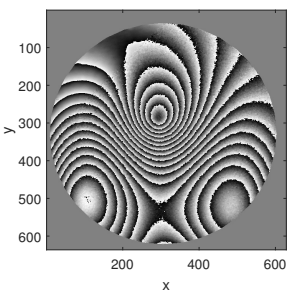

Fig. 5. (a) Phase fringe pattern recorded in a digital holographic interferometry setup corresponding to the out-of-plane displacement of a square aluminum plate. The fringes are obtained within a circular area illuminated by the laser beam. (b) A manually generated binary mask (c) unwrapped phase estimate (in radians) computed using the proposed method with line-scanning approach and (d) re-wrapped phase estimate.

Whereas the inappropriate selection of window width in the PhaseLa method resulted in an inaccurate unwrapped phase estimation, the proposed method provided accurate unwrapped phase estimate as can be observed from the re-wrapped estimate shown in Fig. 3(d).

In order to demonstrate the ability of the proposed method in performing quality guided phase unwrapping, two noisy wrapped phase maps are simulated as shown in Figs. 4(a) and (b). Whereas the phase fringe pattern in Fig. 4(a) carries random phase noise over a certain region, the phase discontinuity is inherent in the true phase map corresponding to the phase fringe pattern in Fig. 4(b). The unwrapped phase estimates shown in Figs. 4(c) and (d) are obtained using the LKF based quality guided phase unwrapping method. The re-wrapped form of these estimated phases shown in Figs. 4(e) and (f) substantiates the applicability of the proposed method.

Figure 5(a) shows a phase fringe pattern recorded in a digital holographic interferometry setup corresponding to the out-of-plane displacement measurement of a non-uniformly clamped square aluminum plate. It can be seen from the figure that the fringes are contained in the circular area illuminated by the laser beam. This area was manually selected using a binary mask shown in Fig. 5(b). The proposed method was implemented using the line-scanning approach. The unwrapped and re-wrapped phase estimates are shown in Figs. 5(c) and (d), respectively.

Another example of phase unwrapping from an experimentally recorded phase fringe pattern is shown in Fig. 6. Figures 6(a) and (b) show, respectively, the object image, and the phase fringe pattern corresponding to the deformation measurement of a fiber reinforced concrete specimen obtained using phase shifting single beam holographic interferometery. Based on the intensity level of the light beam scattered by the object surface and recorded by the CCD camera, a binary mask is generated to separate the bright field associated with the object surface from the dark background. Since the phase fringe pattern carries information on the discontinuous surface displacement profile caused by the crack propagation, we apply a path following
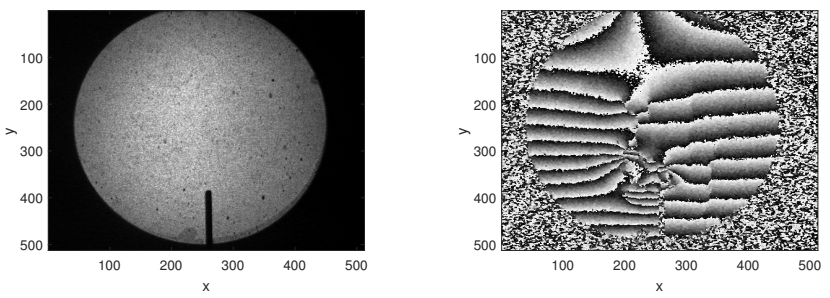

(a)

(b)

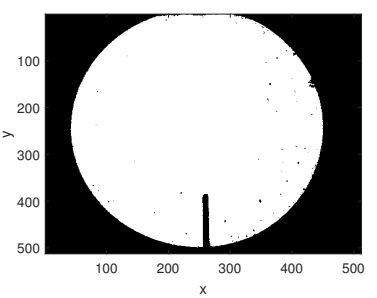

(c)

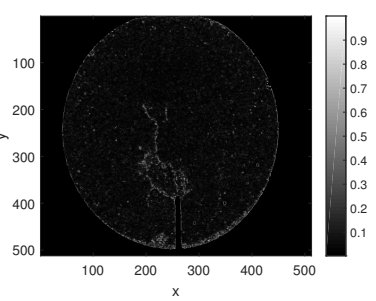

(d)

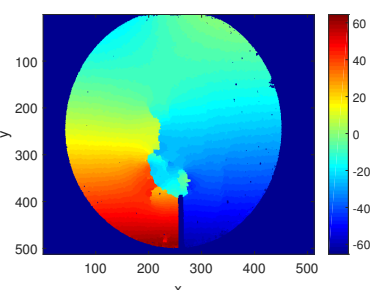

(e)

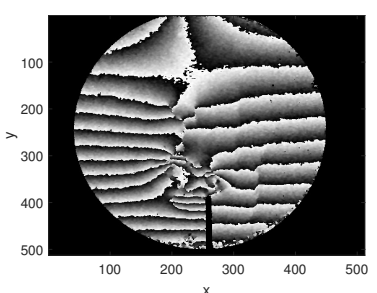

(f)

Fig. 6. (a) Object image (b) wrapped phase (c) binary mask (d) normalized phase derivative variance based phase quality map (e) unwrapped phase estimate (in radians) and (f) re-wrapped phase estimate.

phase unwrapping. A normalized phase derivative variance based phase quality map is computed as shown in Fig. 6(d). Note that a high value of phase derivative variance at given pixel in Fig. 6(d) actually indicates low phase quality at that pixel. The proposed method based on the LKF with quality guided pixel selection approach is applied to the masked phase fringe pattern with $L=1$ to obtain the unwrapped phase estimate shown in Fig. 6(e). The re-wrapped phase estimate in Fig. 6(f) indicates that the unwrapped phase estimate is obtained without any error propagation even in the presence of multiple phase discontinuities.

\section{DISCUSSION}

In the implementation of the proposed method, we have selected $\hat{\boldsymbol{C}}_{0}^{+}=[\phi(x, y), 0,0]$ at the first pixel. The state vector initialization of subsequent pixels is performed using the already estimated state at its highest quality neighbor pixel. In order to make the algorithm withstand different types of fringe variations and noise levels, overestimates of $\hat{\boldsymbol{P}}_{0}^{+}=\operatorname{diag}([100,10,10])$ and $R_{l}=200$ are considered at each pixel, where, the function $\operatorname{diag}(\cdot)$ defines a diagonal matrix with its diagonal elements given by its argument vector. However, in principle, initialization of the error covariance matrix and noise covariance should vary pixel to pixel. An adaptive selection of their values may improve the accuracy of phase unwrapping albeit at an increased computation cost. Note that these settings remain the same in obtaining all the simulation and experimental results provided in the paper. It is important to note that phase unwrapping methods proposed in $[26,29]$ require the estimate of local fringe frequencies to be com- 
puted a-priori using some suitable method. The errors in the estimated local frequencies may propagate in the estimation of unwrapped phase. On the other hand, the proposed method is devoid of this problem because the local frequencies are simultaneously estimated along with the phase. Although we have used phase derivative variance based phase quality map, a different quality map can be used depending on the measurement technique and application at hand. Similarly, a different computationally efficient guiding strategy [13] can also be utilized.

The computation time can be significant in handling large sized phase fringe pattern, especially with the implementation of quality guided fringe scanning strategy. However, utilization of computationally efficient strategy and implementation with a lower level programming language such as $\mathrm{C}, \mathrm{C}++$, etc., can help in reducing noticeably the computational burden.

Some concerns may also arise while handling phase fringe patterns containing phase discontinuities. In the presence of phase discontinuity within the analysis window around a certain pixel, the phase may not be approximated reliably as first order polynomial of spatial coordinates. Although the use of small size window may circumvent this problem to a certain extent, a more robust scheme needs to be developed to address this issue in the future research.

\section{CONCLUSION}

The proposed phase unwrapping algorithm is found to be noise robust and capable of providing continuous and discontinuous unwrapped phase distributions using either the line scanning or a quality guided approach. The adaptive window selection based on the local fringe density provides higher accuracy in phase unwrapping compared to that using a fixed window method or the adaptive window selection based PhaseLa method. The phase unwrapping results obtained using the wrapped phase measurement based linear Kalman filter are equivalent with the traditional nonlinear Kalman filter, however, with an improved computational efficiency. The fusion of quality guided pixel selection and Kalman filter based state estimation provides noise robust phase unwrapping in the presence of phase discontinuities.

\section{APPENDIX}

Figure 7 shows the flow chart of the proposed phase unwrapping algorithm.

\section{REFERENCES}

1. H. Xia, S. Montresor, R. Guo, J. Li, F. Olchewsky, J. . Desse, and P. Picart, "Robust processing of phase dislocations based on combined unwrapping and inpainting approaches," Opt. Lett. 42, 322-325 (2017).

2. S. Wu, L. Zhu, S. Pan, and L. Yang, "Spatiotemporal three-dimensional phase unwrapping in digital speckle pattern interferometry," Opt. Lett. 41, 1050-1053 (2016).

3. B. Osmanoglu, F. Sunar, S. Wdowinski, and E. Cabral-Cano, "Time series analysis of insar data: Methods and trends," ISPRS J. Photogrammetry and Remote Sensing 115, 90-102 (2016).

4. M. Jenkinson, "Fast, automated, n-dimensional phase-unwrapping algorithm," Magnetic Resonance in Medicine 49, 193-197 (2003).

5. X. Su and Q. Zhang, "Dynamic 3-d shape measurement method: A review," Opt. Lasers Eng. 48, 191-204 (2010).

6. D. C. Ghiglia and M. D. Pritt, Two-dimensional phase unwrapping : theory, algorithms, and software (J. Wiley \& Sons, New York, 1998).

7. E. Zappa and G. Busca, "Comparison of eight unwrapping algorithms applied to fourier-transform profilometry," Opt. Lasers Eng. 46, 106-116 (2008).

8. A. M. S. Maallo and P. F. Almoro, "Numerical correction of optical vortex using a wrapped phase map analysis algorithm," Opt. Lett. 36, 1251-1253 (2011).

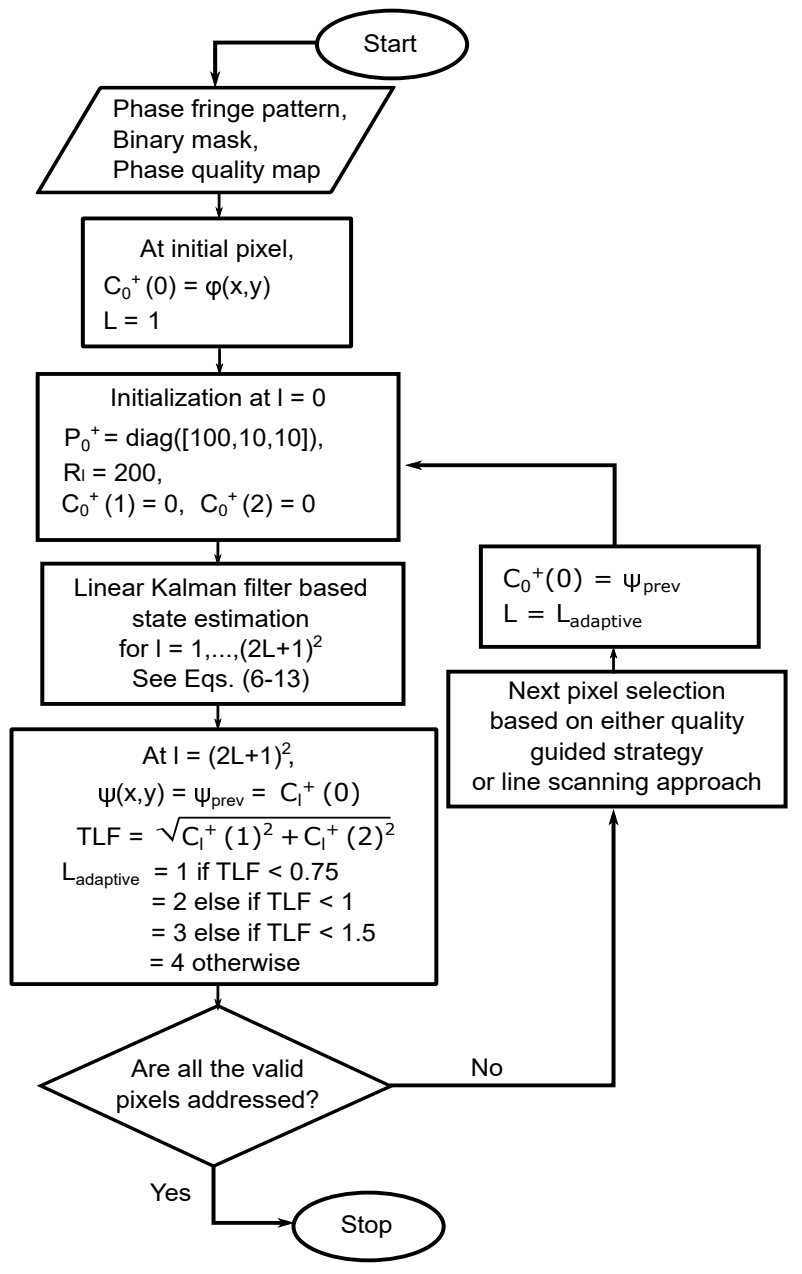

Fig. 7. Flow chart of the proposed phase unwrapping algorithm.

9. R. Cusack, J. M. Huntley, and H. T. Goldrein, "Improved noise-immune phase-unwrapping algorithm," App. Opt. 34, 781-789 (1995).

10. D. Zheng and F. Da, "A novel algorithm for branch cut phase unwrapping," Opt. Lasers Eng. 49, 609-617 (2011).

11. S. A. Karout, M. A. Gdeisat, D. R. Burton, and M. J. Lalor, "Twodimensional phase unwrapping using a hybrid genetic algorithm," App. Opt. 46, 730-743 (2007).

12. J. C. De Souza, M. E. Oliveira, and P. A. M. Dos Santos, "Branch-cut algorithm for optical phase unwrapping," Opt. Lett. 40, 3456-3459 (2015).

13. M. Zhao, L. Huang, Q. Zhang, X. Su, A. Asundi, and Q. Kemao, "Qualityguided phase unwrapping technique: Comparison of quality maps and guiding strategies," App. Opt. 50, 6214-6224 (2011). Cited By :62.

14. X. Su and W. Chen, "Reliability-guided phase unwrapping algorithm: A review," Opt. Lasers Eng. 42, 245-261 (2004).

15. S. Zhang, X. Li, and S. . Yau, "Multilevel quality-guided phase unwrapping algorithm for real-time three-dimensional shape reconstruction," App. Opt. 46, 50-57 (2007).

16. Q. Kemao, W. Gao, and H. Wang, "Windowed fourier-filtered and quality-guided phase-unwrapping algorithm," App. Opt. 47, 5420-5428 (2008).

17. D. C. Ghiglia and L. A. Romero, "Robust two-dimensional weighted and unweighted phase unwrapping that uses fast transforms and iterative methods," J. Opt. Soc.Am. A 11, 107-117 (1994).

18. D. C. Ghiglia and L. A. Romero, "Minimum Ip-norm two-dimensional phase unwrapping," J. Opt. Soc. Am. A 13, 1999-2013 (1996).

19. X. Wang, S. Fang, and X. Zhu, "Weighted least-squares phase unwrapping algorithm based on a non-interfering image of an object," App. 
Opt. 56, 4543-4550 (2017)

20. Z. Zhao, H. Zhao, L. Zhang, F. Gao, Y. Qin, and H. Du, "2d phase unwrapping algorithm for interferometric applications based on derivative zernike polynomial fitting technique," Meas. Sci. Tech. 26 (2015).

21. Y. Guo, X. Chen, and T. Zhang, "Robust phase unwrapping algorithm based on least squares," Opt. Lasers Eng. 63, 25-29 (2014).

22. H. A. Aebischer and S. Waldner, "Simple and effective method for filtering speckle-interferometric phase fringe patterns," Opt. Comm. 162, 205-210 (1999).

23. Q. Kemao, "Two-dimensional windowed fourier transform for fringe pattern analysis: Principles, applications and implementations," Opt. Lasers Eng. 45, 304-317 (2007).

24. Q. Kemao, L. T. H. Nam, L. Feng, and S. H. Soon, "Comparative analysis on some filters for wrapped phase maps," App. Opt. 46, 74127418 (2007)

25. J. C. Estrada, M. Servin, and J. A. Quiroga, "Noise robust linear dynamic system for phase unwrapping and smoothing," Opt. Exp. 19, 5126-5133 (2011).

26. O. Loffeld, H. Nies, S. Knedlik, and W. Yu, "Phase unwrapping for sar interferometry - a data fusion approach by Kalman filtering," IEEE Tran. on Geoscience and Remote Sensing 46, 47-58 (2008).

27. I. Gurov and M. Volynsky, "Interference fringe analysis based on recurrence computational algorithms," Opt. Lasers Eng. 50, 514-521 (2012).

28. R. G. Waghmare, D. Mishra, G. R. K. S. Subrahmanyam, E. Banoth, and S. S. Gorthi, "Signal tracking approach for phase estimation in digital holographic interferometry," App. Opt. 53, pp. 4150-4157 (2014).

29. X. M. Xie and Y. H. Li, "Enhanced phase unwrapping algorithm based on unscented Kalman filter, enhanced phase gradient estimator, and path-following strategy," App. Opt. 53, pp. 4049-4060 (2014).

30. Z. Cheng, D. Liu, Y. Yang, T. Ling, X. Chen, L. Zhang, J. Bai, Y. Shen, L. Miao, and W. Huang, "Practical phase unwrapping of interferometric fringes based on unscented Kalman filter technique," Opt. Exp. 23, 32337-32349 (2015).

31. S. S. Gorthi and P. Rastogi, "Windowed high-order ambiguity function method for fringe analysis," Rev. Sci. Instru. 80 (2009).

32. S. S. Gorthi and P. Rastogi, "Phase estimation in digital holographic interferometry using cubic-phase-function based method," J. Mod. Opt. 57, 595-600 (2010).

33. G. Rajshekhar and P. Rastogi, "Phase estimation using a state-space approach based method," Opt. Lasers Eng. 51, 1004 - 1007 (2013).

34. G. Rajshekhar and P. Rastogi, "Fringe demodulation using the twodimensional phase differencing operator," Opt. Lett. 37, 4278-4280 (2012).

35. J. Langley and Q. Zhao, "Unwrapping magnetic resonance phase maps with chebyshev polynomials," Magnetic resonance imaging 27, 1293-1301 (2009).

36. B. Friedlander and J. M. Francos, "Model based phase unwrapping of 2-d signals," IEEE Tran. on Sig. Proc. 44, 2999-3007 (1996).

37. R. Kulkarni and P. Rastogi, "Local-polynomial-approximation-based phase unwrapping using state space analysis," App. Opt. 56, 29-34 (2017).

38. M. Servin, F. J. Cuevas, D. Malacara, J. L. Marroquin, and R. RodriguezVera, "Phase unwrapping through demodulation by use of the regularized phase-tracking technique," App. Opt. 38, 1934-1941 (1999).

39. V. Katkovnik, J. Astola, and K. Egiazarian, "Phase local approximation (phasela) technique for phase unwrap from noisy data," IEEE Tran. on Image Proc. 17, 833-846 (2008).

40. K. V. Mardia and P. E. Jupp, Directional Statistics (John Wiley \& Sons, Inc., 2008).

41. J. Traa and P. Smaragdis, "A wrapped Kalman filter for azimuthal speaker tracking," IEEE Sig. Proc. Lett. 20, 1257-1260 (2013).

42. R. G. Waghmare, R. K. S. S. Gorthi, and D. Mishra, "Wrapped statisticsbased phase retrieval from interference fringes," J. Mod. Opt. 63, 13841390 (2016)

43. D. Simon, Optimal State Estimation: Kalman, $H$ Infinity, and Nonlinear Approaches (Wiley-Interscience, 2006).

44. S. Montresor and P. Picart, "Quantitative appraisal for noise reduction in 